\title{
Le rôle de l'administration du Travail dans la reconnaissance de la représentativité des organisations professionnelles.
}

Interprétation et usages de l'ex-article L. 133-2 du Code du travail (1946-2006)

The role played by the Labour administration in the recognition of professional organisations. Interpretation and uses of the L. 133-2 Labour Code ex-article (1946-2006)

Jean-Pierre Le Crom

\section{(2) OpenEdition}

\section{Journals}

Édition électronique

URL : http://journals.openedition.org/travailemploi/5790

DOI : $10.4000 /$ travailemploi.5790

ISSN : 1775-416X

Éditeur

DARES - Ministère du Travail

Édition imprimée

Date de publication : 15 septembre 2012

Pagination : 119-136

ISSN : 0224-4365

Référence électronique

Jean-Pierre Le Crom, « Le rôle de l'administration du Travail dans la reconnaissance de la représentativité des organisations professionnelles. », Travail et Emploi [En ligne], 131 | 2012, mis en ligne le 01 décembre 2014, consulté le 30 avril 2019. URL : http://journals.openedition.org/ travailemploi/5790 ; DOI : 10.4000/travailemploi.5790 


\title{
Le rôle de l'administration du Travail dans la reconnaissance de la représentativité des organisations professionnelles. Interprétation et usages de l'ex-article L. 133-2 du Code du travail (1946-2006)
}

\author{
Jean-Pierre Le Crom $\left.{ }^{*}\right)$
}

\begin{abstract}
Cet article s'intéresse au rôle de l'administration du Travail dans l'appréciation de la représentativité des organisations patronales et des organisations syndicales de salariés n'appartenant pas aux cinq grandes confédérations. À partir d'archives inédites, il décrit l'évolution du cadre bureaucratique des enquêtes de représentativité depuis 1936. Il analyse ensuite l'appréciation souple des cinq critères en vigueur jusqu'en 2008 (les effectifs, l'indépendance, les cotisations, l'expérience et l'ancienneté du syndicat, l'attitude patriotique pendant l'Occupation) et les pratiques différenciées selon qu'il s'agit d'organisations d'employeurs ou de salariés. Il met enfin l'accent sur la "doctrine» de l'Administration qui mêle objectivité (la norme des $5 \%$ qui s'est imposée depuis le début des années 1980 ) et subjectivité (le rôle présumé que les organisations reconnues représentatives joueront dans le dialogue social).
\end{abstract}

L'article L. 2121-2 du Code du travail prévoit que «s'il y a lieu de déterminer la représentativité d'un syndicat ou d'une organisation professionnelle autre que ceux affiliés à l'une des organisations représentatives au niveau national, l'autorité administrative diligente une enquête. L'organisation intéressée fournit les éléments d'appréciation dont elle dispose». Jusqu'à la recodification de 2008, l'article L. 133-3 employait une formule un peu différente : «S'il y a lieu de déterminer la représentativité d'une organisation autre que celles affiliées à l'une des organisations représentatives au plan national, le ministre chargé du travail diligente une enquête. L'organisation en cause est tenue de fournir les éléments d'appréciation dont elle dispose». Entre les deux formulations, la différence majeure est que l'expression «le ministre chargé du travail» a été remplacée par «l'autorité administrative». Mais cette différence n'en est pas vraiment une dans la mesure où l'article R. 2121-2, issu d'un décret du 7 mars 2008, prévoit que «les enquêtes relatives à la détermination de la représentativité sont diligentées par le ministre chargé du travail. Pour les professions agricoles, ces attributions sont exercées en accord avec celui-ci par le ministre chargé de l'agriculture».

(*) CNRS (UMR 6297 Droit et changement social, Maison des sciences de l'Homme Ange-Guépin); jean-pierre.le-crom@ univ-nantes.fr
L'objectif de cet article est d'examiner comment l'Administration a mené à bien cette mission que lui a confiée le législateur en 1982. C'est en effet avec l'article 8 de la loi du 13 novembre 1982, l'une des lois Auroux, que la pratique déjà ancienne des enquêtes de représentativité a été légalisée. Leur origine remonte à la mise en œuvre de la loi du 24 juin 1936 et plus particulièrement aux dispositions prévoyant l'extension des conventions collectives à l'ensemble des entreprises dans le champ d'application territorial et professionnel concerné. Parmi les conditions nécessaires à cette extension figurait la signature des «organisations syndicales les plus représentatives». Le rôle de l'Administration, et notamment du bureau des conventions collectives, le 4 e bureau de la Direction générale du travail et de la main d'œuvre (DGTMO), dirigé alors par Melle Renée Petit, est très important. Après avoir reçu une demande d'extension de la part d'une ou plusieurs parties signataires ou du ministre du Travail, le bureau des conventions collectives fait paraitre au Journal officiel un avis demandant aux organisations professionnelles intéressées de se prononcer sur l'extension. Il examine ensuite la demande au prisme des avis reçus et d'une enquête menée par un inspecteur du travail. Le ministre peut alors choisir de ne pas étendre la convention ou demander un complément d'enquête. S'il décide de l'étendre, le dossier est alors transmis au Conseil national économique (CNE), qui fait examiner la demande par la section professionnelle intéressée, puis par la 
commission permanente, et émet un avis. La décision définitive revient au ministre.

Dans cette procédure complexe, l'enquête menée par l'inspecteur occupe une place essentielle. Les rédacteurs du $4 \mathrm{e}$ bureau et les membres des sections professionnelles du CNE ne connaissent pas la situation locale et prennent leurs décisions en fonction de principes généraux. L'inspecteur du travail, qui a très souvent présidé les réunions de la commission mixte lors de l'élaboration de la convention collective, la connaît au contraire très bien. Il n'est dès lors pas étonnant que c'est essentiellement sur le rapport transmis par l'inspecteur que se fondent les fonctionnaires de la DGTMO pour formuler un avis favorable ou défavorable à l'extension (MACHU, 2011, p. 557). Il est important de noter qu'à cette époque-là l'enquête est systématique, même si elle n'est prévue par aucun texte. Suspendue à partir du déclenchement de la Seconde Guerre mondiale, la procédure d'extension sera ensuite simplifiée, puis remplacée en 1941 par une autre procédure qui permet au ministre d'étendre une convention, même si celle-ci n'a pas été signée par les organisations syndicales les plus représentatives (1) (LE CROM, 1995, p. 342).

Après la Seconde Guerre mondiale, l'importance d'une appréciation sérieuse et argumentée de la représentativité s'accroît. Les organisations syndicales voient s'étendre leurs attributions bien au-delà de la seule négociation collective : elles participent aux organes dirigeants des sociétés nationalisées, fournissent en grand nombre les délégués du personnel et les élus des comités d'entreprise et disposent de sièges dans les multiples comités et conseils consultatifs qui sont créés à ce moment (Bockel, 1965). Par ailleurs se pose le problème du pluralisme syndical. Alors qu'un projet de fusion avait été adressé à l'automne 1944 à la Confédération française des travailleurs chrétiens (CFTC) par la Confédération générale du travail (CGT), le départ des militants de la tendance Force ouvrière de la CGT en 1947 va au contraire aboutir à un morcellement du syndicalisme français. Outre la CGT, la CFTC et la CGT-FO existent aussi la Confédération générale des Cadres (CGC), la Confédération générale des syndicats indépendants (CGSI), la Confédération autonome du travail (CAT), la Confédération nationale du travail (CNT, anarchiste) et de multiples syndicats ou fédérations autonomes non confédérés, au premier rang desquels se trouve la Fédération de l'éducation nationale (FEN).

Ce double phénomène d'institutionnalisation et de pluralisme syndical va amener les pouvoirs publics à réfléchir à la fois aux conditions de la représentativité et aux formes de sa reconnaissance. Nous avons déjà traité du premier aspect dans l'introduction de

(1) Circulaire $\operatorname{Tr} 171$ du 21 octobre 1941. ce dossier. Après une tentative ratée d'évaluation de la représentativité sur la base du pourcentage de syndiqués de chaque organisation par rapport à l'ensemble des syndiqués de chaque branche établie par une décision du 13 mars $1947^{(2)}$, une circulaire du 8 avril $1948^{(3)}$, abrogeant la décision précédente, décide qu'en vue de la conclusion des conventions collectives de travail nationales intéressant les grandes branches d'activité sont considérées comme les plus représentatives les organisations syndicales nationales de salariés affiliées à la CGT, la CGT-FO, la CFTC pour l'ensemble des catégories professionnelles, y compris les cadres, et la $\mathrm{CGC}$, en ce qui concerne la seule catégorie des cadres. En outre, en vertu de cette décision, le ministre du Travail se voit conférer le soin d'apprécier le caractère représentatif des organisations syndicales appelées à négocier les chapitres particuliers des conventions collectives nationales de portée générale qui ne visent qu'une catégorie déterminée de salariés, ou des conventions collectives nationales de portée restreinte, et de juger si ce caractère peut être conféré, dans des cas particuliers, à d'autres organisations que celles précédemment visées.

C'est donc sur cette base juridique que les enquêtes de représentativité vont être menées jusqu'en 1983. Leur objectif est de vérifier que les critères édictés par une circulaire Parodi en $1945^{(4)}$, repris ensuite dans la loi du 2 février 1950, puis intégrés dans le Code du travail sous l'article L. 133-2, sont bien respectés. Ces critères sont les suivants : les effectifs, l'indépendance, les cotisations, l'expérience et l'ancienneté du syndicat, l'attitude patriotique pendant l'Occupation.

Notre ambition ici sera d'analyser et de comprendre les pratiques de l'Administration dans une perspective d'histoire sociale du droit. Quelles sont les méthodes employées aux fins de vérification de ces critères? Ont-elles évolué dans le temps? Les critères ont-ils tous la même portée ou certains sont-ils plus importants que d'autres? L'analyse de l'Administration est-elle la même selon qu'il s'agit d'organisations d'employeurs ou d'organisations syndicales de salariés?

Ce sujet est inédit. Il existe certes des travaux sur la représentativité, notamment chez les juristes (Arseguel, 1976) ou les sociologues (Bunel, 1997), mais le rôle particulier de l'Administration dans sa reconnaissance n'avait jamais été abordé en tant que tel. La documentation réunie pour le traiter est elle-même inédite. Elle émane essentiellement des dossiers d'archives du bureau des syndicats (aujourd'hui bureau des relations collectives de

(2) JO du 15 mars 1947

(3) Décision du 8 avril 1948 relative à la détermination des organisations appelées à la discussion et à la négociation des conventions collectives de travail, JO du 9 avril 1948.

(4) Circulaire A. Parodi n 9 D. R. P. du 28 mai 1945. 
travail) de la Direction des relations du travail (DRT, aujourd'hui Direction générale du travail) conservés au Centre des archives contemporaines (CAC) de Fontainebleau ${ }^{(5)}$, au bureau des archives des ministères sociaux ${ }^{(6)}$ ainsi que, pour les plus récents, dans les locaux de la Direction générale du travail (DGT), quai André-Citroën, à Paris (7). Particulièrement riche, elle couvre la période 19462006. Elle a été complétée par quelques entretiens avec certains fonctionnaires du ministère du Travail en charge des enquêtes de représentativité depuis les années 1980.

Cette documentation a été consultée en deux temps. Dans un premier temps, n'ont été étudiés, dans le cadre d'une étude sur la construction de la représentativité patronale (LE Crom, MaGGIGERMAIN, 2010), que les dossiers d'enquêtes relatifs à des organisations patronales, notion entendue au sens large car incluant des organisations d'artisans, de commerçants ou de professions libérales. Ce premier ensemble concerne 129 dossiers, consultés de manière systématique, mais certains d'entre eux portent sur l'ensemble des organisations du secteur. Dans un deuxième temps, pour la réalisation de cet article, ont aussi été examinés des dossiers d'enquêtes relatifs à des organisations syndicales de salariés, mais de manière non systématique.

Il est important de souligner dès maintenant, même si nous y reviendrons dans nos développements, que ces enquêtes n'ont aucun caractère de systématicité. Comme les fonds d'archives n'ont pas été classés de la même manière, il s'avère impossible d'en faire une analyse quantitative systématique pour en déterminer la fréquence. Seul le fonds DRT 1993/002, classé dans l'ordre chronologique des enquêtes menées par l'Administration, permet de se faire une idée du nombre d'enquêtes menées chaque année : quatre en moyenne par an de 1984 à 1986, une en 1987; cinq en 1988; quinze en 1989. Le fonds DRT 2006/010, quant à lui, comprend dix-sept dossiers d'enquête sur une période de trois ans (1994-1996). De manière très approximative, on peut estimer - et cette estimation est confirmée par les fonctionnaires de la Direction générale du travail - que le nombre moyen d'enquêtes diligentées chaque année s'élève à quatre ou cinq par an depuis les années 1980.

Ces enquêtes portent principalement sur des organisations patronales, ce qui est logique puisqu'elles ne bénéficient pas, comme les cinq principales confédérations syndicales de salariés, d'une présomption de représentativité. Leur nombre et leur importance sont par ailleurs très variables selon les secteurs et les branches. Elles sont très peu

(5) Fonds 2005/0116 (5 cartons)

(6) Fonds DRT 1992/001 (15 cartons, 1 manquant), 1993/002

(3 cartons), 2006/010 ( 9 cartons).

(7) Fonds DGT (20 cartons). nombreuses par exemple dans le bâtiment, la chimie ou la métallurgie.

En réalité, en matière de négociation collective, il n'y a d'enquête qu'en cas de demande, et il n'existe de demande qu'en cas de divergence ou de conflit sur la composition des commissions mixtes ou paritaires. Pour la période du Front populaire, Laure MAchu a souligné la rareté de ce type de conflits, imputée au fait que la négociation ne s'est pas toujours faite dans le cadre de la loi du 24 juin 1936; que la présence d'autres syndicats ne posait pas vraiment problème tant la CGT était dominante; et que celle des syndicats chrétiens dans certaines branches était admise en raison de leur ancienneté dans la négociation (MACHU, 2011, pp. 272-273).

Aujourd'hui encore, dans de nombreuses branches, la composition des commissions paritaires (ou mixtes) fait l'objet d'un consensus entre organisations de salariés et organisations patronales, et surtout entre les organisations patronales elles-mêmes. Dans les branches de l'industrie traditionnelle, il n'existe souvent qu'une ou deux organisations patronales. L'administration du Travail parle à ce sujet d' «entente mutuelle», mais elle souligne aussi deux tendances fortes qui ébranlent le consensus majoritairement en vigueur du côté patronal. La première est l'évolution de l'économie vers la tertiarisation. Le développement du secteur tertiaire voit apparaître de nouvelles activités, et donc de nouveaux acteurs, soucieux d'organiser les relations professionnelles dans un cadre adapté à leur activité. Ce cas de figure aboutit souvent à l'organisation d'une enquête de représentativité. Ainsi, parmi d'autres, une enquête fut menée en 1995 sur le syndicat des sociétés de services et des conseils en informatique (3SCI) qui conteste le rattachement des sociétés de service en informatique à la convention SYNTEC, qui regroupe trop d'activités disparates et n'a pas les mêmes spécificités que les métiers de l'informatique (code NAF 72) et le «commerce de matériel informatique» (code NAF $516 \mathrm{G})$. La demande d'enquête émane ici du syndicat lui-même dans la perspective de la négociation d'une convention propre aux métiers de l'informatique ou d'un avenant à la convention « commerces de détail de papeterie, fournitures de bureau, de bureautique et informatique et de librairie» (dite convention FNEBI) qui, selon ce syndicat, décrit certains métiers de l'informatique, ce qui n'est pas le cas de la convention SYNTEC (8).

La deuxième évolution tient à la volonté du ministère du Travail d'améliorer depuis les années 1990 la couverture conventionnelle des travailleurs français. Bien que l'Administration ait fixé, à cette époque, un seuil de 10000 à 15000 salariés pour qu'une branche puisse être créée, elle n'a pas vraiment

(8) DRT 2006/010 (3). 
réussi à éviter l'émiettement corrélatif de la négociation collective. Les nouvelles branches, composées souvent d'acteurs disparates, n'ont pas le caractère «monolithique» des plus traditionnelles. La composition des commissions mixtes se révèle donc plus instable, ce qui peut justifier le recours à une enquête de représentativité (entretien téléphonique avec un fonctionnaire de la DGT, 19 juillet 2012).

Après avoir décrit le cadre bureaucratique des enquêtes de représentativité et son évolution depuis 1936, nous nous attacherons à analyser la manière dont l'Administration interprète le contenu de l'article L. 133-2 critère par critère, puis à comprendre plus globalement sa doctrine en la matière.

\section{Le cadre bureaucratique des enquêtes de représentativité}

\section{Une procédure modifiée en $\mathbf{1 9 8 3}$}

Jusqu'en 1983, le déroulement d'une enquête suit le chemin suivant : une demande, émanant le plus souvent du bureau de la négociation collective, arrive au chef du bureau des syndicats DS3. Celui-ci prescrit alors aux services d'inspection $\mathrm{du}$ travail concernés de mener une enquête; s'il s'agit d'une question nationale, c'est la direction régionale d'île-de-France qui est compétente. Une fois l'enquête menée, le bureau DS3 émet un avis, souvent le même que celui de l'inspection du travail, et transmet au directeur des relations du travail qui confirme ou non l'avis du bureau DS3. Quand l'affaire revêt une importance particulière, notamment sur le plan politique, la décision du directeur du travail est transmise au cabinet.

La procédure est modifiée après l'arrivée de la Gauche au pouvoir en 1981 et l'adoption de la loi du 13 novembre 1982 sur la négociation collective. L'enquête de représentativité devient alors une attribution pleine et entière de l'Administration centrale et des fonctionnaires ayant le grade de rédacteur sont spécifiquement dédiés à cette tâche.

De manière générale, l'inspecteur du travail, jusque dans les années 1980, le rédacteur ensuite, sont laissés très libres de leurs méthodes d'investigation. Quelquefois, ils se contentent de renseignements pris au téléphone avec le président ou un responsable de l'organisation; à l'inverse, certaines enquêtes procèdent à des investigations approfondies : ainsi, en 1963, l'inspecteur divisionnaire du travail et de la main-d'œuvre du Nord-Pas-de-Calais fait visiter les 144 lavoirs automatiques et pressings de la région pour savoir s'ils sont affiliés à une organisation professionnelle et laquelle ${ }^{(9)}$.

(9) DRT 1992/001 (dossier 6). Habillement.
Si la plupart du temps des éléments justificatifs sont demandés, la tendance à davantage de rigueur est toutefois manifeste depuis les années 1980, notamment sur le décompte du nombre réel d'adhérents. Les services déconcentrés peuvent ainsi être mobilisés pour vérifier l'exactitude, département par département, des données communiquées par une organisation nationale. Ce sera par exemple le cas pour la branche des pompes funèbres en 1973 (10).

Pour le décompte des effectifs, les informations peuvent être également demandées ou recherchées auprès de l'Insee (très souvent), de l'Unedic, voire de laboratoires de recherche comme le laboratoire d'économie sociale de Paris I, par exemple, dans le cadre d'une enquête sur l'Union nationale des organismes de développement social, sportif et culturel (UNODESC) ${ }^{(11)}$. Dès lors que la représentativité du syndicat intéresse d'autres départements ministériels, l'enquêteur sollicite un entretien auprès d'eux. Ainsi, avec la direction de l'architecture du ministère de l'Équipement pour le Syndicat de l'architure $^{(12)}$ ou avec la direction de la santé (services d'urgence) pour les syndicats d'ambulanciers ${ }^{(13)}$.

D'autres techniques sont plus inattendues. Ainsi, l'Administration procède-t-elle en 1969 par sondage pour savoir où va la préférence de certaines organisations de professions libérales comme l'Association nationale des avocats de France, la Confédération des syndicats médicaux ou le Syndicat national des notaires qui font partie toutes les trois de deux organisations différentes : la Fédération nationale des professions libérales et l'Union nationale des professions libérales ${ }^{(14)}$.

Ces investigations nécessitent souvent beaucoup de temps. Alors que l'article R. 2121-2 prévoit que «le silence gardé pendant plus de six mois par le ministre chargé du travail saisi d'une demande d'enquête vaut décision de rejet», le déroulement de la procédure dure souvent plus longtemps, comme l'explique un fonctionnaire de la DGT :

«Juridiquement, c'est six mois, mais on ne les tient jamais. Mais on devrait. C'est une question d'organisation interne, c'est le fait que les personnes qui font les enquêtes font beaucoup d'autres choses à côté, c'est un travail qui demande quand on s'y plonge plusieurs journées, voire plusieurs semaines à relancer, à recontacter, et que, nous, on le fait par petits bouts. Du coup, à chaque fois, il faut reprendre le truc à zéro, donc, en gros, je dirais une année, douze mois...»

(Un fonctionnaire de la DGT en charge de l'instruction des dossiers de demande de reconnaissance de représentativité. Entretien du 3 juin 2010.)

(10) DRT 1992/001 (10 et 11). Pompes funèbres.

(11) DRT 2006/010 (3). UNODESC.

(12) DRT 1993/002 (1). Syndicat de l'architecture.

(13) DRT 1993/002 (2). Transports sanitaires privés.

(14) DRT 2006/010 (10). Union nationale des professions libérales / Fédération nationale des professions libérales. 
Ce délai de six mois est relativement court lorsqu'il faut instruire un dossier, c'est-à-dire replacer la demande dans un contexte socioprofessionnel dont le fonctionnaire ne connaît que peu de choses. L'instruction se déroule suivant un processus qui va permettre au fonctionnaire de s'imprégner des éléments contextuels :

«Donc, une fois que j'avais étudié les documents du syndicat, il fallait que j'étudie la profession, justement. Quels étaient les effectifs de la profession, comment elle était répartie sur le territoire national, ça, ça demandait de mobiliser les sources de l'Insee, il fallait avoir des références, quoi! Et ensuite, je comparais les éléments, je faisais une note de présentation, de la profession, du syndicat. Et avec cet avis de service, je joignais la proposition de décision ministérielle. Voilà, la plupart du temps, je prenais le temps aussi de recevoir le syndicat qui demandait à être reconnu représentatif.»
(Un fonctionnaire de la DGT [aujourd'hui sur un autre poste], en charge pendant cinq ans de l'instruction des dossiers de demande de reconnaissance de représentativité. Entretien du 3 juin 2010.)

Pour mener à bien leurs enquêtes, les rédacteurs du bureau appelé aujourd'hui Relations collectives du travail (RCT) ne disposent que d'une note de service dont la date nous est inconnue (voir encadré). Cette note détaille les différentes tâches à effectuer par l'agent chargé de l'enquête à différents moments (J. 0, J. + 5, J. + 33, J. + 45, J. + 75). On en retiendra surtout que le rédacteur est invité à contextualiser son enquête en sollicitant des éléments d'information auprès du président de la commission mixte paritaire, des autres organisations syndicales ou patronales signataires ou adhérentes de la convention collective nationale ainsi que la section «négociation» du bureau RCT.

\section{Encadré \\ Synthèse de la note de service détaillant le déroulement d'une enquête de représentativité}

J. 0 : - date de réception de la demande de reconnaissance de représentativité.

J. + 5 : - accuser réception de la demande (modèle de courrier en annexe 1 : délai pour produire les pièces : 30 jours);

- si nécessaire, contacter l'organisation demanderesse afin d'obtenir les renseignements sur la demande et solliciter éventuellement un rendez-vous au cours duquel la présente procédure et ses enjeux seront expliqués;

- informer les autres organisations d'employeurs et de salariés signataires ou adhérents de la CCN concernée et leur demander des éléments sur ce secteur (modèle de courrier en annexe 2 : délai : 28 jours);

- informer la section «négociation » du bureau des relations collectives du travail du lancement de l'enquête et solliciter des éléments sur l'état des négociations dans le secteur, les positionnements des différentes organisations salariés et employeurs (modèle de courrier en annexe 3);

- solliciter des éléments d'information sur le contexte du secteur et le déroulement des négociations auprès du président de commission mixte (par messagerie ou par téléphone) et/ou auprès des services du ministère en charge du secteur (modèle de courrier en annexe 4).

J. + 33 : - si l'organisation demanderesse n'a pas produit les éléments relatifs à sa demande, la contacter par téléphone et lui repréciser que, sous peine de faire parvenir les éléments demandés dans les 10 jours, sa demande ne sera pas traitée dans le délai de 6 mois visé par l'article R. 2121-2 du Code du travail;

- faire le point des réponses reçues des partenaires sociaux et envoyer un second courrier aux organisations qui n'ont pas encore répondu (modèle de courrier en annexe 5; délai : 10 jours).

J. + 45 : - si les délais précités ont été respectés, un mois après la réception de la demande, le dossier peut être instruit;

- en tout état de cause, commencer la synthèse du dossier et la compléter en cours de traitement avec les informations parvenues ultérieurement (modèle de fiche technique en annexe 6);

- solliciter éventuellement un rendez-vous avec l'organisation demanderesse si des éléments ou des points méritent d'être précisés.

J. + 75 : - à l'issue de la rédaction de la fiche technique et de la proposition de décision (modèle de décision en annexe 7), rédiger une synthèse relative au contexte de cette enquête (modèle de courrier en annexe 8);

- préparer une lettre de saisine du ministère de tutelle et/ou du PCM sur la proposition de décision et la joindre au dossier transmis, communiquer la fiche technique et la proposition de décision (modèle de courrier en annexe 9); - soumettre la proposition de décision à la hiérarchie (pièces requises et modèle de note de couverture en annexe 10).

J. + ... : notifier la décision signée au demandeur ainsi qu'aux organisations de salariés et d'employeurs du secteur (modèle de document en annexe 11). 


\section{Un cadre qui déborde la négociation collective}

Bien qu'instituées dans le cadre de l'extension des conventions collectives, les règles relatives à la représentativité vont servir - et servent encore - à de multiples autres usages. Les enquêtes peuvent également être diligentées dans le cadre d'agréments d'accords collectifs de retraite complémentaire, souvent locaux ou régionaux, ou, dans les années 1950, pour la composition de commissions chargées d'évaluer les temps d'exécution de certains travaux de confection à domicile (éponges en matière plastique, cravates, parapluies, mouchoirs en soie, etc.).

Bien qu'aucun texte ne le prévoit formellement, elles servent aussi à apprécier la représentativité d'organisations siégeant ou demandant à siéger dans certaines instances paritaires ou consultatives, qu'elles soient interprofessionnelles (Conseil supérieur des classes moyennes, Conseil national du crédit, Conseil national de la statistique, caisses d'allocations familiales des travailleurs indépendants, etc.), professionnelles (organisme de prévention professionnel du bâtiment et des travaux publics, commission de la carte d'identité des journalistes professionnels, commission professionnelle du taxi parisien, etc.), nationales, régionales, départementales ou locales. Un certain flou règne sur les règles appliquées ici par chaque ministère de tutelle, même si la référence à la composition de la Commission nationale de la négociation collective est rappelée régulièrement par le Gouvernement. ${ }^{(15)}$

Enfin, de nombreuses enquêtes sont menées dans le cadre de l'agrément de conventions médicales ou paramédicales entre l'État et certaines professions (dentistes, médecins, secteur sanitaire et social, cliniques privées, etc.). Elles sont aujourd'hui encadrées par un dispositif particulier prévu par le Code de la Sécurité sociale (art. L. 162-33, Code de la sécurité sociale) (Arseguel, 1981; Chauchard, 2002).

Les services concernés ne sont donc pas toujours ceux du Travail. La demande d'enquête peut en effet émaner du ministère de la Santé, de l'Éducation nationale, de la Culture, d'organismes de sécurité sociale, voire de la préfecture de police de

(15) DRT 2006/010 (12). Lettre du Premier ministre Pierre Mauroy aux ministres et secrétaires d'État, 14 novembre 1983. Alerté par le ministre des Affaires sociales et de la solidarité nationale sur le fait que, dans certains cas, pour « la composition de certaines instances officielles telles que conseils nationaux, commissions nationales, conseils supérieurs, etc. [...] la nomination des représentants des organisations professionnelles et syndicales ne respecte pas les règles de représentativité de ces organisations au niveau national», Pierre Mauroy demande aux ministres et secrétaires d'État de se conformer à la composition de la Commission nationale de la négociation collective dont la composition a fait l'objet du décret $n^{\circ} 83-461$ du 8 juin 1983 et, en cas de difficulté ou de doute, de consulter le ministre des Affaires sociales et de la solidarité nationale avant toute désignation de représentants dans ces conseils et comités.
Paris. Qui diligente alors l'enquête et qui décide de la reconnaissance ou non de la représentativité? La réponse à ces questions n'est pas évidente. Dans un courrier daté de 1979, le directeur des relations du travail explique :

«il n'appartient pas [...] à l'Administration du travail de se prononcer sur le caractère représentatif d'une organisation syndicale afin de lui permettre de participer aux différents organismes institués dans une profession considérée, par des textes particuliers émanant de diverses autorités administratives et mettant en cause la représentativité de syndicats, sous des formes et selon des conditions spécifiques.

C'est à l'autorité concernée, lors de la mise en œuvre de la procédure de constitution dont elle a la charge de décider, à cette occasion, si telle ou telle organisation syndicale remplit les conditions de représentativité requises au regard du texte instituant ces organismes.

Les services du travail [...] se bornent à transmettre ces éléments à la dite autorité, à qui il appartient, en définitive, de prendre une décision pour l'application des textes la concernant ${ }^{(16)}$.»

En 1994, une note de la DRT semble aller dans le même sens :

«Il n'entre pas dans la compétence du ministre du travail de se prononcer sur l'ensemble des questions de représentativité susceptibles de se poser aux diverses administrations dans le cadre de la mise en place d'organismes consultatifs placés sous leur autorité. En effet, la compétence du ministre du travail en la matière, définie aux articles L. 133-1 et s. du Code du travail, est limitée aux seules questions de représentativité concernant les organisations syndicales d'employeurs et de salariés appelées à négocier des conventions collectives de branche, représentativité qu'il apprécie uniquement par rapport au champ d'application de ladite convention collective.

En dehors de ce cadre, il appartient à chaque département ministériel d'apprécier directement, au regard des critères généraux posés par la loi en matière de négociation collective mais appliqués par la jurisprudence dans d'autres matières, la capacité des interlocuteurs à siéger dans l'organisme consultatif concerné, compte tenu, sauf mécanisme dérogatoire, de son objet et de son assise territoriale, c'est-à-dire selon la règle de concordance $»(17)$.

Ces mises au point sont rendues nécessaires en raison du flou que révèlent les pratiques. Ainsi, en 1989, face à la demande de reconnaissance de représentativité de l'Union syndicale du personnel navigant technique pour participer aux réunions de la commission mixte de la convention collective dont dépendent ses adhérents, le ministre du Travail estime que l'enquête devrait être diligentée

(16) DRT 1992/001 (12). Lettre de Pierre Cabanes au directeur de la Caisse nationale d'assurance maladie des travailleurs salariés, 22 mai 1979.

(17) DRT 2006/010 (3). Commissions régionales d'attribution des licences d'entrepreneurs de spectacle (1994). 
conjointement par le ministère du Travail et celui de l'Équipement, du logement, des transports et de la mer, mais que c'est à ce dernier, seul, de prendre la décision, ce qu'il a toujours refusé de faire jusqu'alors ${ }^{(18)}$. Pourtant, le directeur de cabinet du ministre de l'Équipement, Jean-Cyril Spineta, dans une lettre qu'il signe au nom du ministre, parle d'« une décision relevant du ministre du Travail » (19).

La situation est plus claire pour le secteur de la Santé où les enquêtes sont menées par la direction régionale des affaires sociales d'Île-de-France : ce fut ainsi le cas en 1982 sur les syndicats de médecins et, en 1987, sur les syndicats de dentistes ${ }^{(20)}$.

\section{Un cadre juridique commun aux organisations syndicales patronales et de salariés}

Historiquement, les règles relatives à la représentativité ont été établies en référence à la situation du syndicalisme ouvrier, même si ce n'est jamais explicite. La circulaire du ministère du Travail du 17 août $1936^{(21)}$ qui explicite ce qu'il faut entendre par l'expression «organisations syndicales patronales et ouvrières les plus représentatives », ne fait jamais mention des organisations patronales alors qu'elle fait plusieurs fois référence aux organisations syndicales de salariés, par exemple pour leur représentation au Conseil supérieur du travail ou dans les commissions départementales de conciliation créées pendant l'été 1936. En réalité, le seul objectif de cette circulaire est de régler le problème de la présence de la CFTC dans les commissions mixtes de certaines branches où elle est bien implantée.

De même, la circulaire du 28 mai 1945 qui énonce pour la première fois les cinq critères qui seront repris dans la loi du 11 février 1950, si elle fait référence au rôle des organisations ouvrières CGT et CFTC dans la Résistance, n'évoque jamais les organisations patronales. L'enjeu était ici aussi, dans un contexte où les syndicats ouvriers se voyaient confier de nouvelles attributions, de trouver un moyen de concilier la représentation des travailleurs avec le principe de la liberté syndicale.

En l'absence de dispositif spécifique aux organisations patronales, l'Administration va néanmoins appliquer l'article L. 133-2 et les critères de représentativité qu'il énumère à ces dernières. Cette assimilation s'effectue avec une certaine souplesse, comme on va le voir maintenant.

(18) DRT 1993/002 (3). Lettre du ministre du Travail (bureau

DS3) au ministre de l'Equipement, $1^{\text {er }}$ août 1989.

(19) DRT 1993/002 (3). Lettre du 28 mai 1990.

(20) DRT 1993/002 (1).

(21) $J O$ du 3 septembre 1936, p. 9392.

\section{L'examen des critères de l'article L. 133-2}

L'examen des critères de l'article L. 133-2 par l'Administration s'effectue sous le contrôle du juge qui adopte une attitude pragmatique, selon les espèces qui lui sont soumises. Quelques principes généraux peuvent toutefois être dégagés de cette jurisprudence dont l'Administration tient le plus grand compte. La représentativité n'étant pas, sauf présomption, une qualité intrinsèque, c'est à l'organisation en cause de faire la preuve de celle-ci en apportant les précisions suffisantes à son appréciation. Par ailleurs, jusqu'à la loi du 20 août 2008, les critères ne sont pas considérés comme cumulatifs, les uns pouvant compenser l'absence des autres (HÉAs, 2011).

Concernant les employeurs, la jurisprudence refuse d'admettre une présomption irréfragable de représentativité au profit des syndicats patronaux adhérant aux organisations faîtières (Mouvement des entreprises de France [MEDEF], Confédération générale des petites et moyennes entreprises [CGPME], Union professionnelle artisanale [UPA]) et établit une distinction entre une représentativité stricte des organisations d'employeurs et une représentativité universelle des organisations de salariés (Сhatriot, 2010).

Ainsi progressivement encadré, l'examen des cinq critères de représentativité énoncés par l'article L. 133-2 fait l'objet d'une inégale attention par l'Administration. Par commodité, on distinguera les critères qualitatifs, rarement disqualifiants, des critères quantitatifs, plus souvent mobilisés.

\section{Les critères qualitatifs}

Dans cette première catégorie, on classera l'attitude patriotique pendant l'Occupation, l'indépendance et l'expérience et l'ancienneté; on laissera en revanche de côté pour l'instant le critère relatif aux cotisations, qui n'en est pas véritablement un, dans la mesure où leur examen a surtout pour objectif de vérifier les effectifs déclarés par les organisations.

\section{Le critère relatif à l'attitude patriotique pendant l'Occupation}

Posé par la circulaire Parodi du 28 mai 1945, il visait à écarter des organisations syndicales les plus représentatives celles qui avaient «conservé soit dans les organismes directeurs, soit parmi leurs employés, des personnes ayant participé à la Charte du travail ou qui ne se sont conformées qu'avec mauvaise grâce aux prescriptions de la loi ». En réalité, l'épuration, plutôt sévère, conduisit à un renouvellement important des cadres syndicaux et très peu de «chartistes » continuèrent à exercer des 
responsabilités syndicales après la guerre, en tout cas dans les rangs ouvriers (LE CROM, 1995).

Quelques-uns, cependant, rejoignirent la Confédération générale des syndicats indépendants (CGSI), créée en 1951 notamment par d'anciens syndicalistes proches de René Belin, ministre du Travail du régime de Vichy du 14 juillet 1940 au 18 avril 1942. Assez curieusement, les notes consacrées à la représentativité de cette organisation et à ses successeurs - la Confédération française du travail (CFT) et la Confédération des syndicats libres (CSL) - ne font presque jamais mention de cette caractéristique pour la leur refuser.

Le problème principal touche la CGC en 1947. Une note très critique explique en effet que plusieurs syndicats ou membres de cette organisation sont issus des organismes de la Charte du travail et que leurs anciens dirigeants ont été membres influents des syndicats uniques ou des comités sociaux ${ }^{(22)}$. Ils ont été, à ce titre, frappés de sanctions par les commissions de reconstitution des organisations syndicales de travailleurs. Cela n'empêche pas toutefois la CGC d'être reconnue représentative au niveau national et interprofessionnel pour la catégorie des cadres en 1947.

$\mathrm{Du}$ côté patronal, la référence au critère de l'attitude patriotique pendant l'Occupation est mobilisée par certaines organisations minoritaires contre d'autres, majoritaires : ainsi dans l'industrie du froid en 1947(23) ou dans le secteur de l'électroménager en $1989^{(24)}$. Mais elle ne semble pas retenue par l'Administration. La seule enquête qui évoque explicitement ce critère pour refuser la représentativité touche le Syndicat national du patronat moderne et indépendant (SNMPI) qui avait connu un certain développement après l'arrivée de la Gauche au pouvoir en 1981. Son président aurait été condamné à mort à la Libération pour s'être engagé dans l'armée allemande, sa peine ayant toutefois été réduite à cinq années d'emprisonnement. Mais ce n'est qu'une des raisons pour lesquelles l'Administration lui refuse la représentativité ${ }^{(25)}$.

\section{Le critère de l'indépendance}

Il était déjà mentionné dans la circulaire du 17 août 1936. On pouvait y lire que «s'il est établi que cette adhésion n'est pas réellement libre, qu'elle s'est effectuée sous la pression ou même sous l'influence de certains patrons, on peut se demander dans quelle mesure un syndicat est qualifié pour discuter avec les employeurs les intérêts professionnels des ouvriers et des employés ». Il s'agissait donc clairement d'écarter de la négociation des

(22) DRT 2006/010 (11). Note sur le caractère représentatif des organisations affiliées à la $\mathrm{CGC}, \mathrm{s}$. d.

(23) DRT 1992/001 (1).

(24) DRT 1993/002 (3)

(25) CAC Fontainebleau, 2005/0116 (5), carton 2 conventions collectives susceptibles d'extension des syndicats de salariés dépendants d'un, voire de plusieurs employeurs, des "syndicats-maison» en quelque sorte, ce critère n'ayant pas d'utilité pour les organisations patronales. De fait, la plupart des rapports d'enquêtes de représentativité portant sur celles-ci indiquent que "s'agissant d'une organisation patronale, ce point est d'ordre secondaire».

La question se pose toutefois dans certains cas particuliers, comme dans les professions libérales dans lesquelles l'indépendance du syndicat généralement majoritaire est contestée par les syndicats minoritaires du fait des relations très étroites du premier avec le Conseil de l'Ordre, ou en raison de son intrication avec l'organisation des salariés. Ainsi, dans le secteur des sociétés de crédit immobilier, l'organisation patronale et l'organisation de salariés possèdent le même siège social et la convention collective est signée par un représentant syndical qui est aussi le trésorier de la fédération patronale (26). L'indépendance peut aussi être mise en doute en cas de suspicion d'ingérence de fabricants de produits dans l'activité d'un syndicat (cas de la société Phytomer vis-à-vis de l'Union nationale des instituts de beauté) ${ }^{(27)}$. L'interlocutrice du bureau enquêteur est en effet la secrétaire de la Confédération nationale de l'esthétique et de la parfumerie (CNEP), une organisation qui rassemble, outre l'Union nationale des instituts de beauté (UNIB), la Chambre syndicale du matériel esthétique et la Chambre syndicale des fabricants de produits esthétiques. Pour le rédacteur en charge de l'enquête, «ces liens développés avec la CNEP peuvent légitimement faire craindre un manque d'indépendance de l'UNIB et une ingérence des fabricants et fournisseurs dans les négociations en cours dans le secteur de l'esthétique».

Il s'agit ici de cas d'espèce dont on ne peut tirer aucune conclusion sur la doctrine de l'Administration. La question se pose différemment dès lors que celle-ci constate une relative confusion entre l'organisation patronale et une entreprise du secteur. La situation est assez fréquente. C'est le cas par exemple en 1989 de la Fédération nationale des syndicats du commerce électronique et de l'équipement ménager (FENACEREM) créée par Philips dans l'électroménager (28); du Syndicat professionnel de la sécurité (PROSECUR) qui, dans le secteur de la sécurité, en 1987, rassemble des entreprises qui emploient environ 9000 personnes, dont 4000 pour la seule société SPS ${ }^{(29)}$; de la Fédération nationale des pompes funèbres dont le président est également PDG de la Société générale des Pompes funèbres, société alors en situation de quasi-monopole qui occupe 5000 des 6000 salariés de la branche en

(26) DRT 1992/001 (2). Enquête de 1972

(27) DGT Mirabeau, carton 9. Enquête de 2002.

(28) DRT 1993/002 (3)

(29) DRT 1993/002 (1) 
$1973^{(30)}$; du Syndicat des casinos modernes de France qui, en 1998, se confond pratiquement avec le groupe Partouche ${ }^{(31)}$; du Syndicat de réseaux et services télécoms qui, en 1999, revendique l'adhésion d'entreprises employant 6000 salariés, dont 5000 pour Bouygues Télécom ${ }^{(32)}$.

Une situation relativement proche est celle où une organisation patronale est dominée par une entreprise et ses sous-traitants. C'est le cas, en 1977, de la Chambre syndicale des industries sous-marines sous contrôle de la COMEX. Pour le syndicat concurrent, «cette Chambre syndicale ne comporte, en dehors de la COMEX, que des sociétés industrielles qui travaillent directement avec elle» ${ }^{(33)}$.

Si elle est donc quelquefois soulevée par l'Administration, la question de l'indépendance n'est toutefois jamais un motif de refus de reconnaissance de représentativité des organisations patronales.

Il semble en aller de même pour les organisations syndicales de salariés. La question aurait pourtant pu se poser s'agissant des syndicats ou fédérations affiliés à la CGSI, puis à la CFT et à la CSL, présentes en particulier dans certaines entreprises de l'industrie automobile comme Peugeot, Simca ou Citroën (FAURE, 1998). Les organisations représentatives ont toujours remis en cause l'indépendance de ces organisations et dénoncé l'instrumentalisation dont elles pouvaient faire l'objet par les directions d'entreprises (CAILle, 1977). Pourtant, dans les nombreuses notes qui leur sont consacrées, il n'est fait qu'une seule fois, et brièvement, mention de cette question. Les rédacteurs de ces notes préfèrent argumenter avec le critère de l'audience, plus objectif, qu'avec celui de l'indépendance, politiquement beaucoup plus sensible. Dans les années 1960 et 1970, des questions écrites (34) et des propositions de lois émanant des milieux gaullistes ${ }^{(35)}$ cherchent, directement ou indirectement, à faire reconnaître la représentativité de la CFT et de la CSL.

\section{Le critère conjoint de l'expérience et de l'ancienneté}

Il est rarement développé pour lui-même. L'ancienneté est facilement reconnue après deux ans d'expérience ${ }^{(36)}$ et, en cas de scission, les syndicats bénéficient de leur activité passée. Sous cette rubrique, c'est bien plutôt son activité, telle qu'elle est présentée dans les documents remis par l'orga-

(30) DRT 1992/001 (10 et 11).

(31) DGT Mirabeau, carton 2.

(32) DGT Mirabeau, carton 3.

(33) DRT 1992/001 (12). Professions diverses.

(34) Question écrite de Jacques Soustelle au ministre du Travail, 7 novembre 1974.

(35) Proposition de loi Bernard Pons et al., $\mathrm{n}^{\circ}$ 1092, JO, 22 mai 1979; proposition de loi Kiffer et Feron, 11 avril 1986.

(36) DRT 1992/001 (1). En 1970, la CFT n'est pas reconnue représentative dans l'industrie laitière parce qu'elle n'a été créée qu'en 1968. nisation enquêtée, qui est retracée : publications, congrès, services aux adhérents, participation à des conseils, comités ou commissions, intensité et régularité des contacts avec les pouvoirs publics, etc.

Sauf cas très particulier, il n'est jamais constaté une absence d'activité telle qu'elle devrait entrâ̂ner un refus de reconnaissance de représentativité. Et d'ailleurs, quel serait le niveau suffisant? En réponse à une question sur l'importance de ce critère, un fonctionnaire répond ainsi :

«Parce que, le problème des critères de représentativité, c'est qu'ils ne sont pas très objectifs ces critères. Influence, activité, expérience, on a de petits éléments, mais où est-ce qu'on place la barre? Influence, est-ce que c'est quand ils ont des rendezvous avec dix institutionnels par an ou deux?»

(Un fonctionnaire de la DGT. Entretien du 2 juillet 2010).

\section{Les critères quantitatifs}

\section{Pour les syndicats de salariés : l'audience}

La présomption de représentativité instituée au profit des organisations affiliées à la CGT, à la CFTC, à la CGT-FO, à la CGC, puis à la CFDT impose de distinguer celles-ci de celles qui ne la possèdent pas.

Pour les premières se pose le problème de la portée de la présomption, c'est-à-dire le fait de savoir si elle est irréfragable ou non. Sur ce sujet, l'Administration n'est pas unanime. Deux notes datant du début des années 1970 émettent des avis opposés sur ce sujet. Pour Jacques Legrand, directeur général du travail et de l'emploi, «dans le cas de conclusion de conventions collectives nationales, il existe une présomption de représentativité à l'égard des organisations affiliées aux confédérations figurant sur la liste établie par la décision du 8 avril 1948 modifiée par l'arrêté du 31 mars 1966 [mais] cette présomption n'est pas irréfragable. S'il surgit des difficultés, l'Administration, afin de justifier sa décision, fait procéder à des enquêtes, exploite les résultats des élections professionnelles, etc.». Pour le bureau des conventions collectives, à la même période et sur la même question, «il est certain que la présence de celles des organisations syndicales de salariés qui ont été reconnues les plus représentatives au plan national et interprofessionnel est de droit puisqu'elles ont, du fait de cette reconnaissance très générale, vocation à représenter les salariés de toutes les branches d'activité et à tous les niveaux ${ }^{(37)}$.

Une illustration de ces hésitations et de ces incertitudes peut être trouvée dans un dossier d'enquête de représentativité portant sur la Fédération CFTC

(37) DRT 2006/010 (11). 
du bâtiment (38) qui demande en 1976 à obtenir un siège au Comité central de l'apprentissage du BTP, les huit sièges salariés de cet organisme étant alors occupés par la CGT, la CFDT, la CGT-FO et la CGC. La fédération CFTC a-t-elle le droit d'avoir des représentants dans cet organisme du fait de la présomption de représentativité accordée depuis 1948 à sa confédération ou faut-il tenir compte des $0,8 \%$ qu'elle a obtenus lors des élections aux comités d'entreprise dans le BTP pour la lui refuser?

La lecture du dossier montre tout l'embarras du ministère du Travail. Les notes du rédacteur en charge du dossier sont corrigées par de petites notes manuscrites accompagnant celles, plus officielles, tapées à la machine. L'une d'entre elles souligne que «c'est la première fois, à ma connaissance, que dans un courrier officiel, nous dénions à l'une des cinq organisations de l'arrêté de 1966 le caractère représentatif dans une branche professionnelle». Finalement, la lettre adressée au ministre de l'Éducation nationale, à l'origine de la demande d'enquête, signée par le directeur des relations du travail Pierre Cabanes, et avalisée par le cabinet (le ministre lui-même ayant été averti) conclut à la non représentativité de la fédération CFTC dans le bâtiment du fait de son audience insuffisante, mais «en [...] laissant [au ministère de l'Éducation nationale] le soin d'apprécier l'opportunité de faire participer la CFTC aux travaux du Comité central de coordination de l'apprentissage ${ }^{(39)}$.

Toujours est-il que d'assez nombreuses enquêtes de représentativité portent sur l'ensemble des organisations professionnelles, qu'elles soient patronales ou de salariés et, pour ces dernières, présumées ou non représentatives. Les demandes de l'Administration visant à connaître les effectifs de celles présumées représentatives ne sont que très rarement honorées. En 1949, seule la CGC indique le nombre de ses adhérents dans une enquête visant à apprécier la représentativité des syndicats de salariés de la meunerie ${ }^{(40)}$. Même situation en 1983 lors d'une enquête sur les syndicats de gérants des maisons d'alimentation et d'approvisionnement à succursales ${ }^{(41)}$ : la CGC fournit les renseignements sollicités, la CGT-FO fait «une réponse évasive ne permettant pas d'avoir une appréciation quelconque à leur égard »; la CFDT et la CFTC ne répondent pas; quant à la CGT, elle adresse au directeur régional du travail et de l'emploi d'Île-de-France «une lettre fort désobligeante» dans laquelle il est notamment écrit : «Vous nous demandez de faire la preuve de notre représentativité, ce dont nous nous élevons avec force [sic]. En effet, votre exigence consiste en une remise en cause de la représentativité de la CGT alors que celle-ci est reconnue d'office par le

(38) DRT 1992/001 (2)

(39) Lettre du 22 juin 1977.

(40) DRT 1992/001 (1)

(41) DRT 1992/001 (1).
Code du travail»(42). En 1967, lors d'une enquête cherchant à apprécier la représentativité des organisations professionnelles signataires d'un accord de retraite complémentaire pour les métreurs - vérificateurs, la fédération CGT-FO «refuse de donner le nombre de ses adhérents car elle n'y est pas obligée» et l'union CGT indique que c'est impossible parce que «la comptabilité n'est pas tenue par l'Union mais par les syndicats de base». La même année, l'Administration estime de manière plus générale que «les réponses aux demandes d'enquêtes restent imprécises et le refus systématique opposé par les syndicats - qui, en réalité, n'ont que fort peu d'adhérents - de faire connaître leurs effectifs, devient ces derniers temps encore la règle. Il serait peut-être opportun de rappeler par téléphone (pour des considérations de prudence!) au service de la Direction régionale la jurisprudence du Conseil d'État en la matière, notamment l'arrêt du 15 décembre 1954.»

Cet arrêt estime que l'Administration n'a pas commis d'excès de pouvoir en écartant de la liste des organisations syndicales les plus représentatives d'un secteur, un syndicat qui s'est refusé à répondre à la demande de renseignements qui lui avait été adressée par lettre de l'Administration (43).

Plus généralement, le Conseil d'État a reconnu à l'Administration le droit de demander aux différents syndicats la production de tous documents de nature à permettre d'apprécier leur caractère représentatif (arrêts du 19 mai 1954, du 26 novembre 1954 et, donc, du 15 décembre 1954).

Les difficultés rencontrées par l'Administration à évaluer les effectifs amène cette dernière à privilégier le critère de l'audience : «Les informations fournies par les syndicats étant souvent tendancieuses, l'Administration a été amenée à examiner "l'audience" des syndicats. Ce critère est implicitement contenu dans celui des effectifs et il peut s'apprécier à l'aide des résultats des élections professionnelles (études et tableaux comportant les pourcentages des voix obtenues aux élections des comités d'entreprise établis à la suite d'une exploitation mécanographique» (44).

L'audience est primordiale dans l'appréciation de la représentativité des syndicats ou fédérations indépendantes affiliées à la CSL, créée en 1977 en tant qu'émanation de la CFT, elle-même issue d'une scission avec la CGSI. En 1986, la CSL revendique 250000 adhérents, mais l'Administration estime que le nombre de cartes doit plutôt se rapprocher de 100000 «parmi lesquelles il importerait d'isoler les adhésions spontanées de celles rendues plus ou moins obligatoires au moment de l'embauche».

(42) Lettre du 8 août 1983 adressée à M. Claude Chetcuti, directeur régional du travail et de l'emploi d'Île-de-France. (43) Droit social, $n^{\circ} 3$, mars 1955, pp. 224-225.

(44) DRT 2006/010 (11). Note relative à la notion de représentativité, 15 février 1970. 
Le cadre juridique varie toutefois selon que la représentativité est appréciée au niveau national et interprofessionnel, au niveau des branches ou au niveau des entreprises. Au niveau national et interprofessionnel, la reconnaissance de la représentativité est systématiquement refusée à la CSL depuis qu'un arrêt du Conseil d'État avait annulé un décret du 7 janvier 1959 attribuant, pour des raisons essentiellement politiques ${ }^{(45)}$, un siège à la CGSI au sein de la Commission supérieure des conventions collectives en raison de la faiblesse de ses effectifs. En 1986, ce sont les scores obtenus par la CSL, dans le cadre d'une alliance aux élections prud'homales de 1979, ou bien seule en 1982, de même que son absence d'implantation réelle dans l'ensemble des branches d'activité et sur tout le territoire national qui motivent la non reconnaissance de représentativité(46).

C'est également le Conseil d'État qui prive la CFT et la CGSI des trois sièges qui leur avaient été attribués dans les comités économiques et sociaux régionaux d'Alsace, de Champagne-Ardenne et du Languedoc-Roussillon par un décret du 5 septembre 1973 en estimant que «ni par la proportion faible des salariés qu'ils groupent, ni par les autres éléments caractérisant la représentativité, la CSL ne justifie le caractère d'organisation représentative dans les régions en cause ${ }^{(47)}$.

Dans les branches, c'est l'Administration qui décide, sous le contrôle des juridictions administratives. Elle reconnaît ainsi la représentativité de la CFT dans le commerce et la réparation automobile, les industries chimiques, la transformation des matières plastiques, mais la refuse dans le papiercarton, où la CGSI fait $1,16 \%$ et la CFT $0,42 \%$ aux élections aux comités d'entreprise de 1967 et 1968 , et dans la chimie où la CFT obtient un résultat de $1,31 \%$ en 1968 et $2,15 \%$ en 1969 et la CGSI respectivement $0,78 \%$ et $0,85 \%$. La CFTC ne fait que $2,02 \%$, mais le ministère met ici en avant son expérience et son ancienneté pour reconnaître sa représentativité. ${ }^{(48)}$

Dans les entreprises où la CSL possède quelques fiefs $(68,09 \%$ des voix aux élections professionnelles chez Citroën-Rennes; 54,19 \% chez Peugeot Vieux-Condé; $42,57 \%$ chez Talbot-Poissy),

(45) DRT 2006/010 (11). Note de Jacques Chazelle, directeur général du travail de 1963 à 1966, 24 mars 1966 : «Cette décision pourrait être interprétée comme une reconnaissance implicite du caractère représentatif général, mais il convient de signaler qu'aucune trace n'a été retrouvée dans le service des données de fait, touchant à la représentativité de la confédération dont il s'agit, permettant de justifier cette mesure qui, semble-t-il, avait essentiellement un caractère politique.»

(46) DRT 1993/002 (2). Note du bureau DS3 pour le directeur de cabinet, 5 août 1986.

(47) Arrêt du 21 janvier 1977.

(48) DRT 1993/002 (2). Note du 5 août 1986, op. cit.
l'Administration n'intervient pas. C'est le juge d'instance qui décide en cas de contestation ${ }^{(49)}$.

\section{Pour les syndicats d'employeurs : les effectifs}

Le critère des effectifs, associé à celui des cotisations, se révèle primordial dans l'appréciation de la représentativité des organisations patronales par l'Administration, mais leur décompte est souvent un véritable casse-tête pour celle ci :

«S'ils déclarent cent adhérents et que dans les comptes on retrouve le montant de trois cotisations, ça va nous mettre la puce à l'oreille, mais après, le problème des cotisations, c'est qu'elles ne sont pas standards. Elles sont toujours variables, en fonction du chiffre d'affaires de l'entreprise, du nombre de salariés et, sur le nombre d'adhérents, il n'y a que l'organisation elle-même qui demande sa représentativité qui peut nous la donner. Après, on peut avoir des indices au travers des caisses de retraite, des déclarations d'adhérents dans d'autres institutions...»

(Un fonctionnaire de la DGT en charge de l'instruction des dossiers de demande de reconnaissance de représentativité. Entretien du 2 juillet 2010.)

Si les organisations patronales jouent très généralement le jeu et communiquent les renseignements qu'elles possèdent, ceux-ci ne sont pas en effet toujours très fiables. En 1984, les médecins adhérant aux syndicats nationaux de la Confédération des syndicats médicaux de France et ceux de la Fédération des médecins de France sont comptés deux fois; en 2000, la Fédération nationale des pressings revendique 1500 adhérents alors que l'Administration aboutit après enquête à un chiffre de $380^{(50)}$; en 2002, 1'Union nationale des instituts de beauté déclare 350 adhérents payant chacun une cotisation forfaitaire de $750 \mathrm{~F}^{(51)}$. Le montant cumulé des cotisations devrait donc représenter une somme totale de 262500 F. Or la comptabilité indique seulement un montant de $60000 \mathrm{~F}$. Dans certaines professions, notamment libérales, le phénomène des multi-adhésions complique encore le travail des enquêteurs.

Ce travail consiste aussi à vérifier l'implantation véritablement nationale de l'organisation enquêtée. Une organisation présente seulement dans quelques départements ne sera pas reconnue représentative sauf si l'activité à laquelle elle se réfère est concentrée d'un point de vue géographique.

Le nombre d'adhérents ayant été plus ou moins établi, l'Administration doit alors déterminer si le niveau des effectifs est suffisant pour la déclarer représentative. Depuis les années 1980 et jusqu'à aujourd'hui, elle se réfère de manière assez systématique à un taux de syndicalisation de $5 \%$. Ce

(49) Ibid.

(50) DGT Mirabeau, archives vivantes. Enquête d'octobre 2000 .

(51) DGT Mirabeau, carton 9. 
pourcentage, que l'on trouve mentionné dans de très nombreuses enquêtes, est confirmé par plusieurs fonctionnaires de la DGT. L'une d'entre elles explique ainsi :

«Voilà, d'avoir les $5 \%$. Il y avait une jurisprudence qui avait refusé la représentativité quand l'organisation n'atteignait pas les $5 \%$. Donc, on accusait réception de cette demande, et ensuite on lançait une enquête." (Entretien du 3 juin 2010.)

Cette invocation rituelle du seuil de $5 \%$, qui se transmet de génération en génération de rédacteur au sein du bureau chargé de mener les enquêtes de représentativité, ne repose en réalité sur rien. On le trouve certes sous la plume du commissaire Genevois dans les conclusions qu'il livre au Conseil d'État à l'occasion d'un recours de la Fédération des chirurgiens-dentistes de France contre un arrêté du 21 juillet 1977 approuvant un accord passé entre les caisses d'assurance-maladie et la Confédération nationale des syndicats dentaires, accord dont ladite fédération avait été exclue de la négociation ${ }^{(52)}$.

Il y explique que la jurisprudence, qui s'est attachée à définir la notion d'organisation syndicale la plus représentative, "a posé en principe que la notion de représentativité devait s'apprécier en fonction non seulement des effectifs de l'organisation en cause mais aussi des autres éléments caractéristiques à cet égard», le principe s'appliquant aussi bien aux organisations de salariés qu'aux organisations patronales. Il nuance ensuite son propos en soulignant que la jurisprudence du Conseil d'État, «si elle ne confère pas au critère qualitatif un rôle exclusif, lui attache néanmoins une grande importance en ce sens que la faiblesse des effectifs d'une organisation peut constituer un obstacle dirimant à la reconnaissance de sa représentativité». Après avoir cité plusieurs arrêts en ce sens, il ajoute que ceux-ci «se gardent bien de donner des précisions numériques» et ajoute : «Vous indiquez néanmoins que le nombre des adhérents doit être apprécié aussi bien en lui-même que par comparaison avec les autres organisations syndicales représentatives (cf. 11 avril 1962 Confédération française des travailleurs chrétiens Rec. p. 275). On peut déduire également des indications données par vos Commissaires de Gouvernement (cf. en particulier les conclusions de M. Odent sur votre décision d'Assemblée du 27 juin 1951 C. G. T préc.) qu'en deçà d'un seuil de $5 \%$ des effectifs de l'ensemble de la profession considérée un groupement syndical ne saurait en principe être regardé comme représentatif» (Genevols, 1979).

Dans ses conclusions, qui ne sont que des conclusions et non des injonctions, le commissaire Genevois ne fait donc que reprendre à son compte

(52) Fédération des chirurgiens-dentistes de France, 25 mai 1979, Recueil Lebon, 1979, p. 242. celles de l'un de ses prédécesseurs. Le problème est que les conclusions du commissaire Odent sur un arrêt d'Assemblée du Conseil d'État du 22 juin $1951^{(53)}$ n'évoquent pas en réalité ce seuil de $5 \%$, ne concernent pas les organisations patronales et n'ont pas été suivies par le Conseil.

Dans cette affaire, la CGT avait attaqué un décret du 15 avril 1950, pris en application de la loi du 11 février 1950, qui avait enlevé un siège à la CGT au profit de la CGT-FO dans une section de la Commission supérieure des conventions collectives spécialisée pour l'étude des questions intéressant les travailleurs agricoles. Après avoir analysé les arguments en faveur et en défaveur de la représentativité de la CGT-FO auprès des salariés de l'agriculture, le commissaire Odent conclut que «les prescriptions de la loi du 11 février 1950 vous font un devoir de tenir principalement compte des effectifs pour apprécier le caractère représentatif des organisations syndicales et [que] «le pourcentage de $3 \%$ [et non $5 \%$ ] des adhérents de la CGT-FO nous paraît, en définitive, insuffisant pour reconnaître que cette organisation est l'une des plus représentatives des salariés en agriculture» ${ }^{(54)}$.

Le plus surprenant est qu'en réalité les conclusions du commissaire du gouvernement ne sont pas retenues par le Conseil d'État. Celui-ci estime, au contraire, que la CGT-FO avait bien le droit d'obtenir un siège dans la section spécialisée de la Commission supérieure des conventions collectives, «compte tenu du faible effectif des travailleurs syndiqués».

Le seuil de $5 \%$ qui sert de référence aux fonctionnaires depuis le début des années 1980 est donc le fruit d'une erreur d'interprétation. Il ne repose sur aucun texte, ni sur aucune décision de jurisprudence, le Conseil d'État s'étant toujours refusé à préconiser un pourcentage minimal. Rappelons aussi que la seule fois où les pouvoirs publics ont fixé des seuils minima de représentativité, en 1947, ces seuils étaient beaucoup plus élevés puisqu'ils se situaient à $10 \%$ des effectifs syndiqués pour l'ensemble de la branche d'activité et à $25 \%$ des effectifs si l'une seulement des catégories professionnelles était concernée.

Ce taux de $5 \%$ ne s'impose toutefois à l'Administration que comme une norme relative. D'autant plus relative d'ailleurs que les entreprises adhérentes aux organisations patronales ne pèsent évidemment pas toutes le même poids, à la différence des organisations de salariés où un syndiqué vaut un autre

(53) Droit social, 1951, p. 472.

(54) «La composition de la section agricole de la Commission supérieure des conventions collectives et la représentation de Force ouvrière. Arrêt de la section sociale du Conseil d'État, 22 juin 1951, conclusions du commissaire du gouvernement Odent et obs. de M. j. D. V.», Droit social, n² 2, février 1952, pp. 110-115. 
syndiqué. Dans l'électroménager, par exemple, Darty ou Conforama ne pèsent pas la même chose qu'un commerçant indépendant. Représenter $5 \%$ des entreprises ne signifie pas du tout la même chose selon que les dites entreprises sont petites, voire très petites, moyennes ou importantes. Dès lors l'Administration prend en compte d'autres éléments.

La principale variable est celle du nombre d'employés des entreprises adhérentes. Ainsi l'Union nationale des organismes de développement social, sportif et culturel (UNODESC) ne représente que $3 \%$ des effectifs des entreprises de l'animation socioculturelle mais $6 \%$ des salariés ${ }^{(55)}$. En ce qui concerne la Chambre syndicale de la reliure-dorurebrochure (RBD), la faiblesse du taux d'adhésion, situé en dessous du seuil des $5 \%$, est compensée par le fait que, «dans toutes les hypothèses envisagées, les adhérents retenus emploient plus de $5 \%$ des salariés du secteur RBD, sauf à retenir le chiffre de 12000 salariés donné par la Chambre syndicale mais qui est infirmé par toutes les données statistiques officielles » ${ }^{(56)}$.

Quant à la Fédération française des pompes funèbres, elle compte un nombre plus important d'adhérents (116) que la Fédération nationale des pompes funèbres (60), mais elle occupe un nombre de salariés bien moindre (415 contre 6000 ) et n'est donc pas reconnue représentative ${ }^{(57)}$.

L'Administration tient compte également du chiffre d'affaires des entreprises adhérentes au syndicat. Dans l'électroménager, par exemple, le Syndicat national du commerce d'équipement de la maison (SYNCOMEM) a 450 adhérents en 1988, soit $2 \%$ du nombre d'entreprises de la profession, mais les entreprises adhérentes (But, Darty, Conforama) emploient 30000 personnes et ont une part de marché de 9 milliards, soit $21 \%$ du marché national. Il est donc, évidemment, reconnu représentatif(58).

D'autres critères peuvent également être reconnus comme d'autant plus importants qu'ils peuvent être objectivés par des statistiques publiques, par exemple la puissance frigorifique comparée de deux fédérations patronales concurrentes dans l'industrie du froid à la fin des années 1940, puissance mesurable par des renseignements venus du ministère de la Production industrielle ${ }^{(59)}$.

La critère des effectifs dans la reconnaissance de la représentativité des organisations d'employeurs se révèle également prééminent dans la jurisprudence du Conseil d'État qui néglige tous les autres critères (HÉAs, 2011, et les arrêts qu'il cite).

(55) DRT 2006/010 (3). Enquête de 1994.

(56) DRT 2006/010 (3). Enquête de 1996.

(57) DRT 1992/001 (10 et 11). Enquête de 1973.

(58) DRT 1993/002 (3).

(59) DRT 1992/001 (1)
$\mathrm{Au}$ bout du compte, l'examen des critères de représentativité de l'article L. 133-2 par l'Administration présente deux caractéristiques principales. $\mathrm{La}$ première est que, si elle prend le soin, surtout à partir des années 1980, d'examiner chacun des critères de l'article L. 133-2, elle élargit ses investigations à d'autres indicateurs, comme l'activité et l'audience et en privilégie certains, comme les effectifs, pour les organisations d'employeurs, et l'audience, pour les organisations de salariés ne bénéficiant pas de la présomption de représentativité.

La seconde est la prise en compte de la jurisprudence, particulièrement celle du Conseil d'État. Les arrêts, ou même les conclusions des commissaires du gouvernement de ce dernier, quelquefois mal interprétés, inspirent de manière nette les rédacteurs en charge des enquêtes qui transposent allègrement des décisions relatives aux organisations de salariés à des dossiers concernant les organisations patronales, dans des contextes et des situations pourtant très différentes. Cette prise en compte de la jurisprudence du Conseil d'État s'opère toutefois dans une atmosphère de suspicion. Agents et responsables de la DGT estiment ainsi souvent que "les juges n'y comprennent rien» ou qu' «ils sont en aveugle sur ces questions» dans la mesure où «ils ne peuvent mesurer quelles sont les conséquences de faire venir telle ou telle organisation à la table des négociations». Ces commentaires sont tout à fait essentiels pour comprendre ce qui se joue dans la reconnaissance de la représentativité d'une organisation patronale.

\section{La «doctrine» de l'Administration}

Pour l'Administration du travail, l'examen du respect des critères de l'article L. 133-2, qui implique le respect de la loi, aussi vague soit-elle, se conjugue avec le souci de faire fonctionner de la manière la plus efficace qui soit le système conventionnel. Les critiques entendues contre le Conseil d'État portent précisément sur l'aveuglement de ce dernier face à cette mission essentielle de l'Administration du travail. Juge versus Administration, juridicité versus opportunité, règles contre pratiques : la construction de la représentativité patronale se joue autour de ces tensions.

Pour l'Administration du travail, la reconnaissance de la représentativité s'inscrit d'abord dans le cadre de la négociation collective et de la capacité de l'organisation à s'y intégrer sans difficulté. De ce point de vue, l'Administration est d'abord un «facilitateur». En pratique, elle poursuit un double objectif : d'une part, limiter le morcellement de la négociation; d'autre part, améliorer le dialogue social dans la profession. 


\section{Limiter le morcellement de la négociation}

Le nombre de branches professionnelles «connues de la DGT» s'élève aujourd'hui à 687 dont près de 400 qui regroupent moins de 5000 salariés ${ }^{(60)}$. Après le découpage «autoritaire» institué par la loi de 1936 et surtout de 1946, les services du ministère du Travail ont adopté un régime plus souple où la branche d'activité est d'abord ce que les parties ont entendu qu'elle soit (Despax, 1989, p. 474). Mais, en réalité, ce ne sont pas les deux parties à la négociation qui déterminent l'étendue de la branche d'activité qui sert d'assisse à l'extension, ce sont les seuls syndicats patronaux, les syndicats de salariés ne jouant aucun rôle en la matière. Une organisation patronale dissidente, en désaccord avec une convention collective ou un avenant, peut donc créer une nouvelle branche, dès lors qu'elle est représentative dans ce périmètre ${ }^{(61)}$.

Cette situation explique largement l'émiettement de la négociation collective que l'administration du Travail cherche désormais à freiner ou à réduire.

La politique du ministère du Travail vise en effet à limiter le nombre de branches et à favoriser leur regroupement, notamment pour mieux organiser leur suivi administratif. Cette politique a des incidences directes sur la reconnaissance ou la non reconnaissance de représentativité. Les demandes émanent en effet souvent d'organisations qui ne sont représentatives que d'une partie de la branche. Cette situation est bien expliquée par les rédacteurs en charge des enquêtes :

«En plus, on leur explique bien, parce que des fois, il y a des confusions, on leur dit bien que nous, c'est sur l'ensemble de la convention collective et non pas sur un sous-secteur, en ce moment, on a des enquêtes dans le domaine de la santé et du paramédical... C'est pas parce qu'ils sont reconnus par la DSS, la direction de la sécurité sociale ou la direction de la santé, pour participer à certaines négociations liées à leurs professions que nous, on va les reconnaître représentatifs pour la négociation collective. On n'a pas forcément les mêmes critères. Du coup, on leur explique ce qu'on va regarder. On essaye au maximum de ne pas reconnaître des sous-secteurs dans une convention collective.

- Pourquoi?

- Dans ce cas-là, on se retrouverait avec des conventions collectives où il y aurait dix syndicats. On est dans une logique où on est plus dans une fusion des champs d'application, on a des conventions collectives qui regroupent des activités très diverses, et si on imagine que pour chaque activité, il y a un

(60) Chiffres cités par le rapport Poisson (Poisson, 2009, p. 41).

(61) Voir le commentaire de Philippe Langlois (1979) sous l'arrêt du Conseil d'État du 15 décembre 1978 (Chambre syndicale des industries de désinfection, désinsectisation et dératisation), Recueil Dalloz Sirey de doctrine de jurisprudence et de législation, Paris, Dalloz, p. 332. syndicat, on ne va jamais s'en sortir. Parce qu'après, il faut voir les conséquences, nous, quand on reconnaît une organisation sur un champ donné, ça veut dire qu'au moment de l'extension quand cette organisation a signé un accord, si elle signe toute seule, l'accord est quand même étendu à l'ensemble des salariés de ce champ. Or, si c'est une organisation sectorielle, ça ne sera qu'au morceau qu'elle représente. Alors, là, ça fait un bazar, ça morcelle complètement la négociation.»

(Entretien du 2 juillet 2010.)

Ce type de situation se retrouve fréquemment dans les enquêtes de représentativité : le Groupement des entreprises de plasturgie du bâtiment est bien représentatif en 2005 des entreprises de plasturgie du bâtiment (77\%) mais pas de la branche de la plasturgie en général (4\% des entreprises du secteur; $5,1 \%$ des salariés) ${ }^{(62)}$; le Syndicat des fabricants de salaisons du Nord (1969) est bien représentatif de sa branche d'activité spécifique, à savoir la profession des fabricants de salaisons, conserves de viandes, charcuterie en gros, mais il ne peut être considéré comme l'une des organisations d'employeurs les plus représentatives de tout le secteur professionnel de l'alimentation du Nord(63); l'Union des libraires de France (271 adhérents) est bien représentative des 1200 libraires stricto sensu recensés par le bureau du Livre du ministère de la Culture, mais pas des 10309 établissements faisant activité de commerce de détail de livres, journaux et prospectus (les maisons de la presse). Le taux de syndicalisation est évalué à $22,5 \%$ dans le premier cas, à 2,6\% dans le second ${ }^{(64)}$.

Ce schéma vaut aussi pour les syndicats de salariés. Tel est par exemple le cas pour le Syndicat national des mécaniciens au sol de l'aviation civile (SNMSAC), créé en 1968 et qui revendique 786 adhérents au 31 décembre 1987 dont 398 à Air France, entreprise qui n'est pas régie par la convention collective. Le SNMSAC demande en 1988 à participer aux réunions de la commission mixte paritaire de la convention collective des entreprises de transport aérien. Le rédacteur de l'enquête note bien que le syndicat est un interlocuteur habituel de la Direction générale de l'aviation civile, qu'il participe à des jurys de délivrance de diplômes et à plusieurs commissions comme celle du travail constituée en 1974 pour l'élaboration des normes d'entretien des aéronefs, celle pour l'élaboration du manuel d'exploitation des aéronefs et certificats de navigabilité, celle pour la modification de l'annexe 1 de la convention de Chicago (licence des personnels) et qu'il est également représenté dans des commissions du ministère de l'Éducation nationale, notamment pour l'élaboration de CAP ou de BTS. Son activité importante en matière de sécurité lui

(62) DGT Mirabeau, carton non archivé.

(63) DRT 1991/001 (1)

(64) DGT Mirabeau, carton 17. 
vaut également d'être présent au sein des commissions chargées d'élaborer les recommandations de sécurité sur les aéroports. Pourtant, sa demande de reconnaissance de représentativité fait l'objet d'un avis négatif «dans la mesure où le SNMSAC, syndicat "de métier", ne représente qu'une très petite partie des effectifs salariés couverts par la convention». En effet, s'il représente 12,8\% de la population des techniciens aéronautiques, il ne représente que $2,4 \%$ de celle qui relève de la convention collective ${ }^{(65)}$.

La non-reconnaissance de représentativité peut aussi empêcher la signature d'avenants, entraînant ainsi le morcellement de la convention collective. Un bon exemple de cette situation est celui de la branche du commerce et de la réparation de motocycles ${ }^{(66)}$ où il existe deux organisations : la Fédération nationale du commerce et de la réparation du cycle et du motocycle (FNCRM) - 17,86 \% des effectifs employeurs et un peu plus de $39 \%$ des effectifs salariés - dont la représentativité est «incontestable», et la section cycles et motocycles du Centre national des professionnels de l'automobile (CNPA), fondée par des dissidents de la FNCRM dans le seul secteur des deux-roues et qui rassemble moins de $5 \%$ des employeurs de la branche. Cette situation amène le rédacteur chargé de l'enquête à préconiser de ne pas reconnaître la représentativité du CNPA, tout en laissant entendre que ce pourrait être le cas plus tard, compte tenu de son dynamisme.

Cette analyse est contestée par le sous-directeur de la négociation collective : «Dans cette branche conventionnelle, le CNPA joue un rôle de leader incontestable. La non-reconnaissance de sa représentativité en ce qui concerne une sous-partie du champ d'application de la convention collective nationale aura des conséquences importantes sur la cohésion de la branche. En effet, la FNCRM se montre parfois réticente à signer les accords souvent novateurs proposés par le CNPA. Le fait qu'elle soit seule représentative du secteur du cycle et du motocycle a pour conséquence d'exclure ce secteur des accords dont elle n'est pas signataire. Le fait que le CNPA ne soit pas reconnu représentatif va conduire immanquablement à la signature d'avenants qui ne pourront être étendus dans l'ensemble du champ d'application de la convention collective nationale entraînant ainsi un morcellement de cette dernière» ${ }^{(67)}$.

(65) DRT 1993/002 (3). Note sur la représentativité du Syndicat national des mécaniciens au sol de l'aviation civile, 17 juin 1988.

(66) DRT 2006/010 (5). Enquête de 1995.

(67) Ibid. Note du sous-directeur de la négociation collective.

\section{Favoriser le dialogue social}

Ce point de vue du sous-directeur de la négociation collective introduit une deuxième dimension de la «doctrine» de l'Administration : la reconnaissance ou la non reconnaissance de la représentativité dépend aussi des conséquences présumées qu'elle aura sur le dialogue social :

«Le critère des effectifs est privilégié, mais il fait partie d'un "faisceau d'indices" qui seront mobilisés différemment suivant le contexte. Ce qui est important, c'est la capacité dont fait montre l'organisation qui demande à être reconnue représentative à s'insérer dans un processus de négociation collective. L'Administration privilégie cet aspect : l'organisation est-elle volontaire pour s'engager dans des négociations, souhaite-t-elle s'engager de manière constructive? ... Car la représentativité qui est conférée par l'Administration est celle liée à la négociation collective et seulement celle-là.»

(Un fonctionnaire de la DGT en charge de l'instruction des dossiers de demande de reconnaissance de représentativité. Entretien du 24 mars 2010.)

Dès lors qu'un doute existe, ce qui compte est de savoir si la reconnaissance entraînera «un grain de sable dans la négociation collective ou de l'huile dans les rouages», selon l'expression imagée d'un haut fonctionnaire de la DGT. Pour le savoir, des contacts privilégiés sont pris avec les présidents de commissions mixtes paritaires qui transmettent leur connaissance fine des rouages de la négociation et des conditions de leur bon déroulement. Dans le même esprit, l'avis des autres organisations syndicales - et principalement des organisations de salariés dans le cas d'une procédure de reconnaissance d'une organisation d'employeurs - est souvent sollicité. L'objectif est d'abord la reconnaissance mutuelle des organisations :

«Ce qui prime de fait sur la reconnaissance expresse de représentativité, c'est l'entente mutuelle. Dans $99 \%$ des branches, il n'y a pas de décision expresse. Le ministère du Travail rend combien de décisions expresses en matière de représentativité par an? Une poignée qui concerne, en majorité, les employeurs. Du coup, comment fonctionnent les négos dans beaucoup de branches? Sur une reconnaissance mutuelle.»

(Un fonctionnaire du ministère des Transports, président de la Commission nationale d'interprétation et de conciliation.)

Cette volonté de l'Administration est manifeste dans de nombreux dossiers d'enquêtes de représentativité. On n'en prendra que quelques exemples. Créé en 1978, le Syndicat de l'architecture demande à participer aux réunions de la commission mixte paritaire de la convention collective nationale des cabinets d'architectes, signée, côté employeurs, par le conseil de l'Ordre et l'Union nationale des syndicats français d'architectes, et dénoncée en 1985 par ces deux organisations. 
Les critères d'ancienneté, d'indépendance et d'activité étant considérés comme remplis, reste la question des effectifs, a priori insuffisants. Si le Syndicat des architectes revendique 1000 adhérents sur 22000 architectes inscrits à l'Ordre, seuls 174 ont réglé leur cotisation en 2005. Cette faiblesse des effectifs - loin des $5 \%$ retenus par l'Administration - n'empêche toutefois pas la reconnaissance de la représentativité de cette organisation professionnelle :

«Le Syndicat des architectes a une activité et une audience dépassant largement ses effectifs et qui lui a valu d'être reconnu comme interlocuteur dans les domaines qui touchent la profession des architectes. Compte tenu de cette reconnaissance entrée dans les faits, de l'activité déployée par cette organisation syndicale, de la faiblesse du nombre de syndiqués dans la profession, il me paraît possible de reconnaître le Syndicat des architectes comme représentatif au plan national et de le convoquer aux réunions de la commission mixte de la convention collective nationale des cabinets d'architectes.

Une telle décision permettrait une représentation au sein de cette commission des architectes ayant un point de vue différent sur le rôle des architectes, l'organisation et l'avenir de cette profession. Un pluralisme des syndicats d'employeurs toujours souhaitable en matière de négociation collective serait ainsi assuré»» (68).

L'idée dominante est ici d'organiser l'avenir de la profession par le pluralisme des organisations d'employeurs, d'autant que les autres organisations sont considérées comme conservatrices ou frileuses.

Dans le cas du Syndicat des organisateurs et réalisateurs d'actions promotionnelles et commerciales (SORAP), qui fait l'objet d'une enquête en $2004{ }^{(69)}$, c'est la moralisation de la profession qui détermine le ministère à accorder la reconnaissance de représentativité, malgré l'avis initial de l'auteur de l'enquête qui estime que «les critères relatifs à l'expérience et à l'activité ne peuvent être considérés comme suffisamment remplis», le nombre d'adhérents ne pouvant par ailleurs pas être établi. Ici, c'est le cabinet qui prend la décision, comme le montre cet échange de courriels :

Cabinet du ministre, 13 février 2004 : «Où en est l'enquête de représentativité du SORAP qui a été contestée par la seule CGT? Sauf excellentes raisons de votre part, il faut reconnaître cette représentativité, compte tenu des engagements pris par la SORAP pour la moralisation du marchandisage $»$.

DGT, 13 février 2004 : «Nous accélérons l’enquête, mais nous butons sur un premier problème qui est celui de la détermination des effectifs, il est très difficile d'avoir des données ».
Cabinet du ministre, 13 février 2004 : «Je sais bien. Par définition, vu le secteur mais je répète qu'il faut reconnaître leur représentativité sauf si vous avez les éléments contraires. J'attends donc le dossier d'ici la fin du mois».

Cette politique n'est pas nouvelle. En 1972, déjà, le ministère du Travail reconnaît la représentativité de la Fédération des employeurs de personnel employé de maison (FEPEM), qui ne regroupe pourtant qu' $1,5 \%$ des employeurs de la branche, dans un souci de protection des salariés du secteur : «La pratique constante de notre Département consiste à étendre très libéralement, chaque fois que cela est possible, celles des clauses des conventions collectives qui permettent de garantir au personnel intéressé une protection effective minimale en matière notamment de salaire et de condition de travail. Par contre, ont toujours été exclues de l'extension les clauses qui auraient entraîné, pour les employeurs, des charges ou obligations allant au-delà des prescriptions habituelles de la réglementation» $(70)$.

Dans tous les cas, la reconnaissance de la représentativité de l'organisation patronale est liée à la volonté des pouvoirs publics de promouvoir la négociation collective, d'améliorer le sort des salariés ou de moraliser la profession. À l'inverse, l'Administration peut refuser la reconnaissance de représentativité à une organisation dont elle estime que l'entrée dans une commission mixte risquerait de perturber un dialogue social jugé satisfaisant dans la profession.

En 1993, une enquête portant sur le Syndicat national des entrepreneurs de nettoyage, dont les effectifs approchent les $5 \%$ de la profession, conclut à la non reconnaissance immédiate, «l'entrée de ce syndicat dans les négociations n'étant pas souhaitable car risquant de déséquilibrer les négociations qui se déroulent sans difficulté avec la Fédération des entreprises de propreté ${ }^{(71)}$.

Du côté des salariés, le même type d'argument est employé vis-à-vis de l'Union syndicale du personnel navigant technique qui demande à participer en 1989 aux réunions de la commission mixte paritaire dont ses adhérents dépendent. L'Administration refuse la reconnaissance de représentativité «pour éviter les cris et hurlements du principal syndicat concurrent, le syndicat national des pilotes de ligne ${ }^{(72)}$.

La nécessaire bonne tenue des réunions de commissions mixtes est aussi l'argument utilisé par l'Administration pour ne plus convoquer la CFT aux réunions de celle de l'industrie des contreplaqués.

(70) DRT 1992/001 (1). Lettre du directeur général du travail et de l'emploi au directeur de cabinet du ministre, 4 septembre 1972.

(71) DRT 1993/002 (2)

(72) DRT 1993/002 (3). Note manuscrite, s. d.

(68) DRT 1993/002 (1). Enquête
(69) DGT Mirabeau, carton 10. 
Bien que reconnue représentative de cette branche en 1971, sa présence est néanmoins contestée par les autres syndicats et les incidents se multiplient. Saisi par la CFT, le tribunal administratif de Paris lui donne raison: «Les circonstances que des incidents dus à la présence de la CFT lors des réunions précédents aient eu lieu n'est pas de nature à motiver légalement le refus de la convoquer aux commissions mixtes de la branche»(73). Une nouvelle enquête est donc diligentée, mais l'Administration annonce d'avance que ses résultats ne changeront rien à la situation : "Nous devons procéder à cette enquête pour tenir compte du jugement du T. A. de Paris. [...] Mais des résultats positifs pour la CFT n'entraîneraient pas nécessairement sa convocation : en fait nous continuerions d'ajourner la commission mixte... ${ }^{(74)}$.

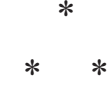

On retiendra quatre idées principales de cette enquête sur le rôle de l'Administration dans la reconnaissance de la représentativité syndicale.

La première est la distinction qu'il est nécessaire d'opérer entre la négociation collective et la participation des organisations aux autres instances paritaires et consultatives. Une organisation peut tout à fait être reconnue suffisamment représentative dans un secteur pour participer aux divers comités, conseils et commissions qui existent (par exemple dans le transport aérien) et, en même temps, ne pas l'être pour participer aux réunions de la commission mixte paritaire, parce qu'elle n'a pas assez d'adhérents ou qu'elle ne représente qu'une partie de la profession.

Le deuxième élément est que l'enquête n'a aucun caractère de systématicité. Dans la plupart des branches, la participation des syndicats aux négociations collectives est le résultat d'une reconnaissance mutuelle, sans intervention de l'Administration. Celle-ci n'intervient qu'en cas de litige, de contestation ou de contentieux entre organisations. L'enquête de représentativité est donc bien la fille du pluralisme syndical, dont on ignore trop souvent qu'il est la règle dans de nombreuses branches, aussi bien pour les organisations patronales que pour les organisations de salariés.

Le troisième élément à retenir est qu'en matière de négociation collective, l'Administration évalue la représentativité avec une certaine souplesse. Si le pourcentage de $5 \%$ des effectifs de la profession s'est imposé depuis le début des années 1980, il ne vaut que de manière relative. Le jugement se veut en réalité aussi prospectif et tient compte des conditions concrètes du dialogue social dans les branches, de ce que l'arrivée d'un nouveau syndicat peut lui apporter de souffle nouveau ou, au contraire, des difficultés qu'elle est susceptible de véhiculer.

Le quatrième élément porte sur la différence entre les organisations patronales et les organisations de salariés. La plupart des enquêtes portent sur des organisations d'employeurs parce qu'il existe une présomption de représentativité au profit des syndicats, unions et fédérations appartenant aux cinq grandes confédérations de salariés. Pour autant, il existe aussi des organisations non confédérées et il a existé des confédérations de syndicats indépendants ou autonomes qui devaient prouver leur représentativité, à l'instar des organisations d'employeurs, sur la base de l'article L. 133-1. L'examen différencié des critères de représentativité par l'Administration à l'intérieur d'un cadre juridique commun (centré sur l'audience pour les syndicats de salariés et sur les effectifs pour les syndicats d'employeurs) ne doit pas surprendre. Il est le fruit d'un positionnement très différent des deux catégories d'organisations dans les relations professionnelles en France. Les syndicats d'employeurs sont beaucoup plus ancrés sur le service aux adhérents que les syndicats de salariés; l'analyse de leur activité ne saurait donc être appréhendée de la même manière. Il existe par ailleurs une forte différence du point de vue de l'audience, qui peut facilement être mesurée pour les organisations de salariés, via les élections professionnelles, et pratiquement pas pour les organisations d'employeurs. Si les pouvoirs publics souhaitent rapprocher les régimes de représentativité pour tenir compte de la loi du 20 août 2008, cela passera nécessairement par l'instauration d'un dispositif de mesure fiable de l'audience des organisations patronales (MAGGI-GermaIN, 2011). 


\section{Bibliographie}

Arseguel A. (1976), La notion d'organisations syndicales les plus représentatives, thèse de doctorat en droit, Université de Toulouse 1, 2 vol., 698 p.

Arseguel A. (1981), «La représentativité des syndicats dans les conventions médicales», Droit social, $\mathrm{n}^{\circ} 4$, avril, pp. 349-356.

BocKel A. (1965), La participation des syndicats ouvriers aux fonctions économiques et sociales de l'État, Paris, LGDJ, 622 p.

BunEl J. (1997), «Représentation patronale et représentativité des organisations patronales », Travail et emploi, $\mathrm{n}^{\circ} 70$, pp. 3-19.

Caille M. (1977), Les truands du patronat, Paris, éditions sociales, 1977, $305 \mathrm{p}$

Chatriot A. (2010), «Quelle représentativité pour les organisations patronales?», in Daumas J.-C. (dir.), Dictionnaire historique des patrons français, Paris, Flammarion, pp. 1006-1009.

Chauchard J.-P. (2002), «À propos de la convention médicale : réflexions informelles sur la représentativité des organisations syndicales de médecins», Travail et Protection sociale, $\mathrm{n}^{\circ}$ 6, juin, pp. 7-8.

Despax M. (1989), Droit du travail. 7, Négociations, conventions et accords collectifs, Paris, Dalloz, 2e éd., $586 \mathrm{p}$.

FAVRe D. (1998), Ni rouges, ni jaunes : de la CGSI à la CSL, l'expérience du syndicalisme indépendant de 1945 à nos jours, Midi moins le quart, $299 \mathrm{p}$.
Genevois B. (1979), «Sur l'enquête de représentativité», Conseil d'État, 25 mai 1979, Conclusions du commissaire du gouvernement, Droit social, $\mathrm{n}^{\circ}$ 9-10, septembreoctobre, pp. 364-368.

HÉAS F. (2011), «La représentativité des organisations salariées et patronales en droit français », Revue de droit du travail, $\mathrm{n}^{\circ} 2$, p. 9197.

Le Crom J.-P. (1995), Syndicats, nous voilà. Vichy et le corporatisme, Paris, éditions de l'Atelier, $410 \mathrm{p}$.

Le Crom J.-P., Maggi-Germain N. (dir.) (2011), La construction de la représentativité patronale, rapport pour la Dares, avril, $400 \mathrm{p}$.

Machu L. (2011), Les conventions collectives du Front populaire. Construction et pratiques du système français de relations professionnelles, thèse de doctorat d'histoire, université Paris-Ouest Nanterre-La Défense, 2011, 943 p. Consultable sur: http://bdr.u-paris10.fr/theses/ internet/2011PA100158.pdf

Maggi-Germain N. (2011), «La représentativité des organisations professionnelles d'employeurs",, Droit social, $\mathrm{n}^{\circ} 11$, novembre, pp. 1072-1082.

Poisson J.-F. (2009), Rapport sur la négociation collective et les branches professionnelles. Rapport au Premier ministre, Paris, La Documentation française, coll. «Collection des rapports officiels». Rapport consultable à l'adresse : http://www.ladocumentationfrancaise.fr/var/ storage/rapports-publics/094000216/0000.pdf 